\title{
A Method of Inspection Feature Evaluation Based on DMIS
}

\author{
Guijiang Duan ${ }^{1, a}$, Ye Ren ${ }^{2, b}$ \\ ${ }^{1}$ School of Mechanical Engineering and Automation, Beihang University, Beijing, 100191, China \\ ${ }^{2}$ School of Mechanical Engineering and Automation, Beihang University, Beijing, 100191, China \\ aemail: greatd@163.com, bemail:ren_ye@163.com
}

Keywords: Evaluation; Inspection Feature; DMIS

\begin{abstract}
Evaluation of inspection features mostly depends on manual operation, and thus lead to time-consumed and error prone. A new method of feature evaluation based on DMIS is put forward in this paper. The typical types of features and evaluations are summarized. The process of feature evaluation based on DMIS is introduced. Finally, by a case study, the method of feature evaluation based on DMIS is proved to be feasible and applicable.
\end{abstract}

\section{Introduction}

Feature evaluation is one of the essential means to measure the machined part as a qualified or unqualified product. In general, inspectors acquire the evaluation results through the professional measuring software and equipment. For a CMM [1, 2] (Coordinate Measuring Machine), the matching software may be PC-DMIS [3], RationalDMIS [4] or Virtual DMIS [5]. First, Inspectors manually operate the CMM to collect some features which need to be inspected. And then define the tolerances of the inspected features. Finally, evaluate the features to the tolerances by means of some specific functions in the measuring software.

Disadvantages of evaluating features by operating measuring equipment and software are as following: a) High labor intensity for CMM inspectors; b) Time-consumed, and thus lead to occupy too much machine-hour; c) Error prone when increase in the number and kinds of inspected parts. Therefore, this paper puts forward a new idea of feature evaluation, which is based on DMIS [6, 7].

\section{DMIS Reference}

DMIS is an acronym for Dimensional Measuring Interface Standard, which was developed by IIT Research Institute under contract to CAM-I (Computer Aided Manufacturing International, Inc), and completed in March 1986. The purpose of DMIS is to resolve the intercommunication of measuring data and the unification of data form between different measuring equipments.

In DMIS, inspection features are classified into nineteen kinds, such as arc, circle, cone, closed ended parallel, cylinder, ellipse, generic point, generic curve, geometry feature, generic surface, line, user created object, feature with opposite parallel planes(slot or block), pattern, plane, point on the edge of a surface, right rectangular, sphere, and torus. Tolerance types are divided into seven categories. Fig 1 (a) (g) separately shows the dimensional tolerance, form tolerance, orientation tolerance, location tolerance, runout tolerance, profile tolerance and other tolerance. Feature evaluation is based on features and tolerances.

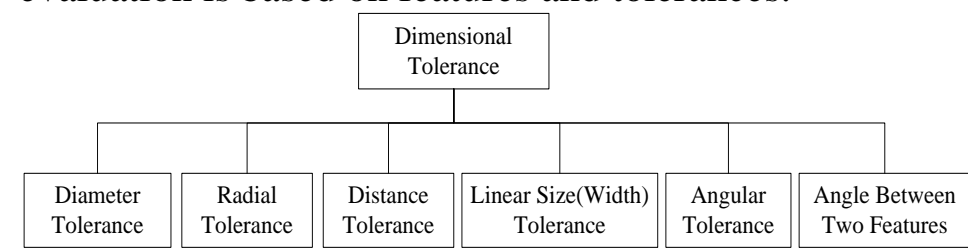

(a) Dimensional tolerance

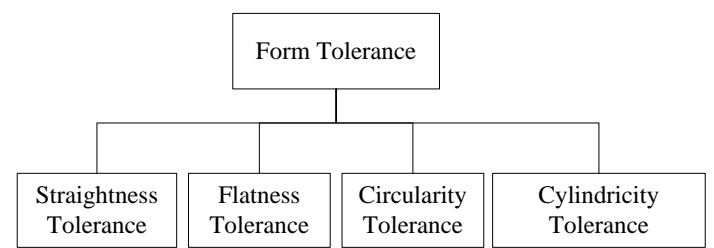

(b) Form tolerance 


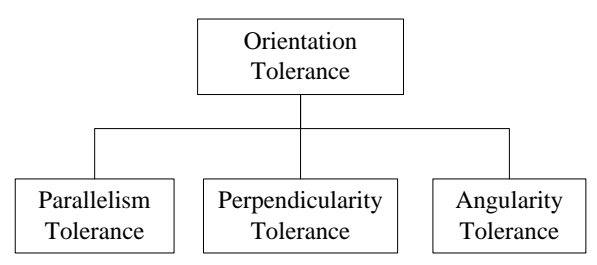

(c) Orientation tolerance

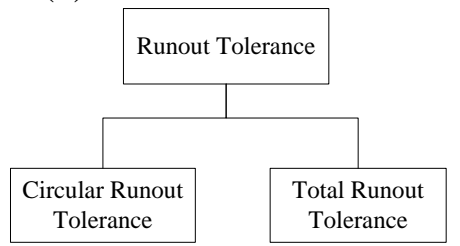

(e) Runout tolerance

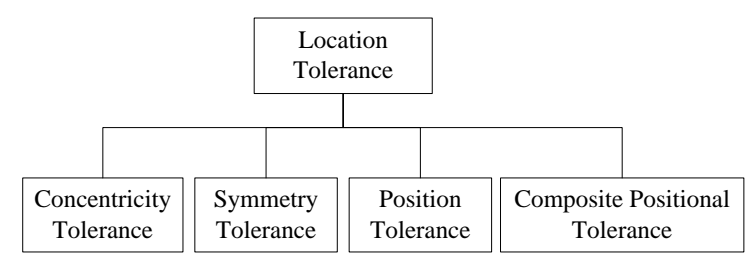

(d) Location tolerance

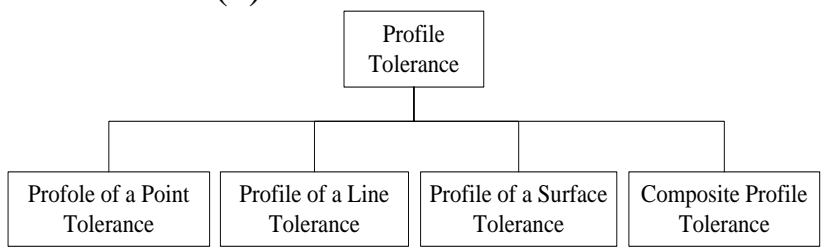

(f) Profile tolerance

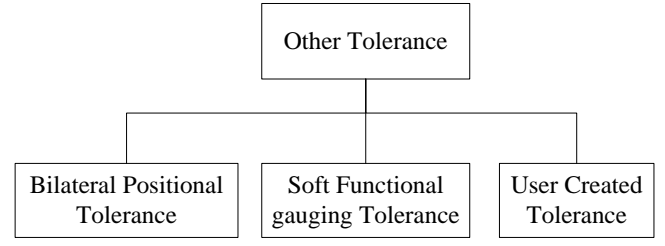

(g) Other tolerance

Fig.1. Evaluation types

DMIS is not only a standard, but also a computer language and a data format. DMIS language with some specific format can establish the relationship between inspecting feature(s) and tolerance(s), and thus acquire the evaluation results.

\section{Feature Evaluation}

Feature evaluation is to evaluate the measured feature to the tolerance which is previously defined based on DMIS. Fig 2 shows the process of feature evaluation.

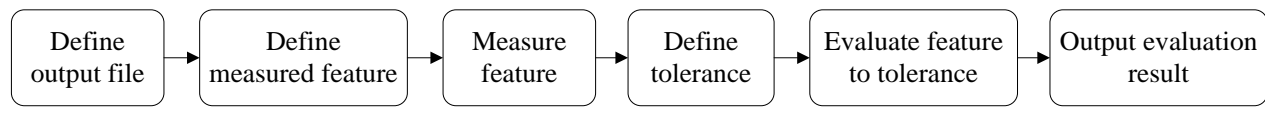

Fig 2 Process of feature evaluation

Output file can be classified into two categories, those controlled by the DME (Dimensional Measuring Equipment) and those controlled by the user. The user file(s) to which output data will be sent, or from which input data is received, is control by the DEVICE, OPEN, WRITE, and CLOSE statement. The DEVICE statement identifies that it's a storage device. The OPEN statement opens the previously defined storage device and establishes the connection's input/output attributes. The WRITE statement is used to transfer data from the program to the user defined file. The CLOSE statement can be used to close files opened with the OPEN statement. In this paper, user defined output file is used, and evaluation result is passed to the file by WRITE statement.

Follow are additional examples of user defined output files. They demonstrate how to output evaluation result by use of DEVICE, OPEN, WRITE and CLOSE statement.

DID(userdata) = DEVICE/STOR, 'D:Iuserfile.txt'

OPEN/DID(userdata), DIRECT, APPEND

WRITE/DID(userdata), 'Evaluation result'

\section{CLOSE/DID(userdata), END}

Measured feature Definition is controlled by definition statements which describe the detailed information for specific case of geometry with CAD data. All the feature types have been introduced in the part of "DMIS Reference". They must occur before the feature is measured. A definition statement describes one specific set of conditions and assigns a label name to it for easy reference. The label name is then used in another statement to refer to the object defined. According for defining a plane, the DMIS statement can be "F(PLN_1) = FEAT/PLANE, CART ,0, 0, 1, 0, 0, $20 ”$. 
Feature measurement is controlled by the MEAS statement which signifies that a measurement of a feature is to be performed. The measurement sequence begins with a MEAS statement and ends with the ENDMES statement. In the following example, four points are set in order to measure a plane called "PLN_1".

MODE/PROG, MAN

$\mathrm{F}\left(\mathrm{PLN} \_1\right)=$ FEAT/PLANE, CART, 0, 0, 20, 0, 0, 1

MEAS/PLANE, F(PLN_1), 4

PTMEAS/CART, -10, 10, 20, 0, 0, 1

PTMEAS/CART, 10, 10, 20, 0, 0, 1

PTMEAS/CART, 10, -10, 20, 0, 0, 1

PTMEAS/CART, -10, -10, 20, 0, 0, 1

ENDMES

The MEAS statement signifies that a plane called PLN_1 is to be measured by taking four point measurements. The following statements specify that point measurements will be used, the location of the point to taken. The ENDMES statement signifies the end of the sequence.

Tolerance definition in DMIS is generic, in that there is no pointer to the feature to which the tolerance is applied. The tolerance definition need not occur in a DMIS file immediately following the feature definition, but the tolerance definition must occur before evaluation. According for defining flatness tolerance, the DMIS statement can be "T(TOL_1) = TOL/FLAT, 0.1". 0.1 is the number representing the width of the tolerance zone defined by two parallel planes within which the surface of the feature lies.

Feature evaluation according to tolerance can be executed by two DMIS statements. One is EAVL statement, and the other is OUTPUT statement. The difference between two statements is that EVAL statement is only used to generate a tolerance actual, but OUTPUT statement causes the evaluation data to be output. In this paper, EVAL statement is used to obtain the evaluation result, because different equipment has different output format.

\section{Case Study}

A case of evaluating a plane's flatness based on DMIS is given here to demonstrate and validate the method introduced above. The principle of other features evaluation is similar. Fig 3 shows the process of evaluating a plane to a flatness tolerance based on DMIS.

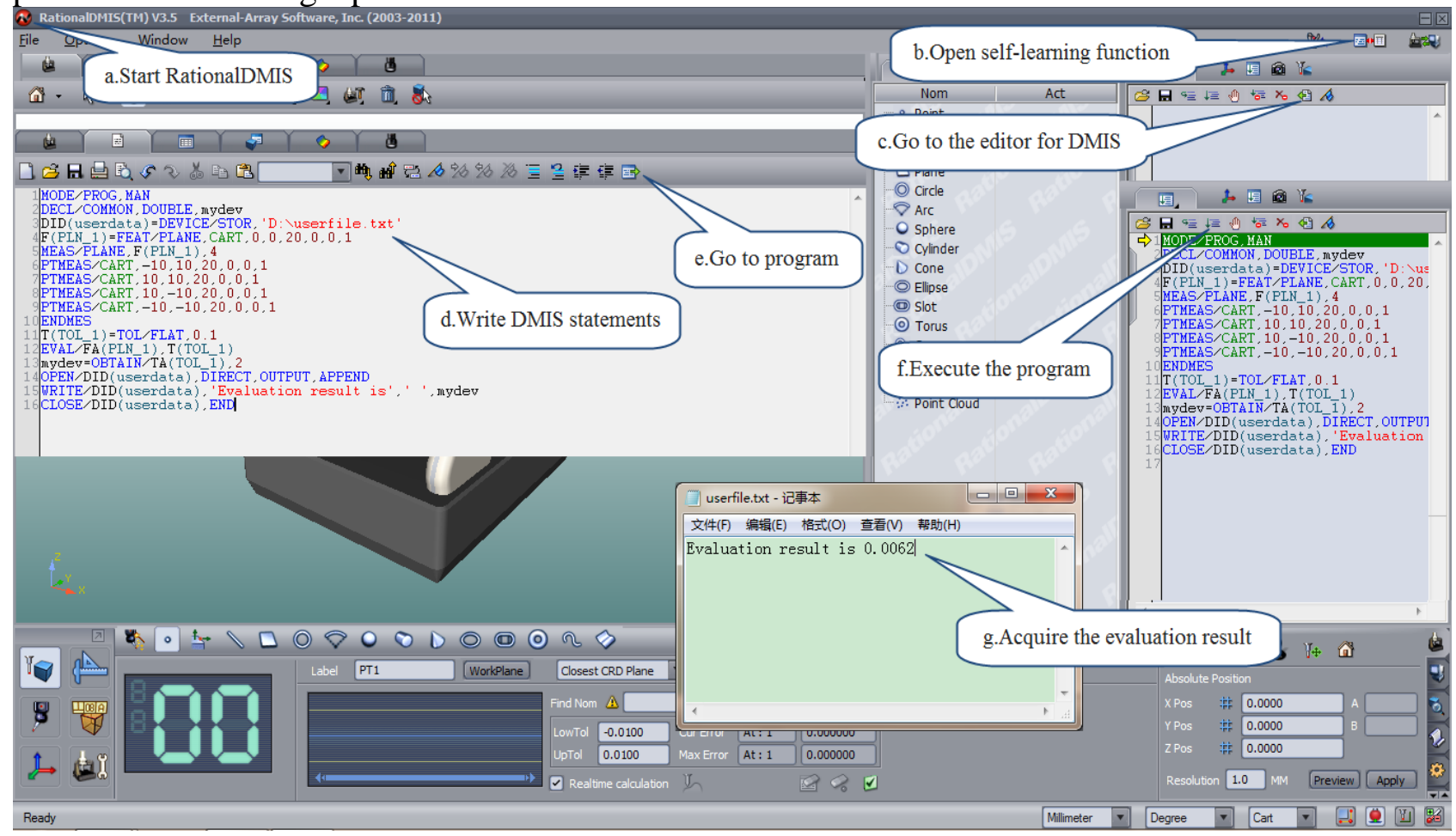

Fig 3 Process of evaluating a plane’s flatness 
The evaluation steps are list as followings:

Step a: Start the RationalDMIS which is one professional CMM measuring software and developed by External-Array Software Incorporated.

Step b: Open the self-learning function that is a button on the upper right of the interface, and then the DMIS program can be seen.

Step c: Go to the editor for DMIS in which we can write DMIS statements.

Step d: Paste or manually write DMIS statements into the editor or directly import a DMI file.

Step e: Go to the Program, that is, transfer the DMIS statements to program code which can be executed by RationalDMIS.

Step f: Execute the program from the first line.

Step g: The user defined output file is created, and evaluation result is written into the file.

\section{Conclusion}

By the above case study, the method of feature evaluation based on DMIS is proved to be feasible. With the increase of inspect tasks, features evaluation directly by DMIS will be a necessity. One of the main advantages of the method is that inspect program can be edited and debugged before the hardware in place, respecting to the shortage of measuring equipments. The DMIS program between different measuring software can be shared, so as to improve the efficiency and accuracy of product inspection.

\section{References}

[1] Martin, Ian. The evolution of CMM probing systems [J]. Journal of Manufacturers' Monthly, 2014: 20-21.

[2] MORGAN, MARTIN. The Complete CMM [J]. Journal of Quality, 2013, V52 (2): 22-35.

[3] Danford, Matthew D. High-Accuracy CMM with PC-DMIS Software [J]. Journal of Modern Machine Shop, 2006, V79 (4): 220-222.

[4] External-Array Software, Inc. RationalDMIS user manual [M]. 2005.

[5] Virtual DMIS Supports Neutral CAD File Format [J]. Journal of Quality Progress, 2002, V35 (5): 109.

[6] Zhang Lin. The Application of DMIS to Coordinate Measuring Technology [D]. Xi'an: Xi'an University of technology, 2006.

[7] ANSI/CAM-I 104.0-2001, Part 1. Dimensional Measuring Interface Standard Revision 04.0 [S]. 2001. 